\title{
Analysis of cytokine mRNA expression in syngeneic islet grafts of NOD mice: interleukin 2 and interferon gamma mRNA expression correlate with graft rejection and interleukin 10 with graft survival
}

\author{
A. Rabinovitch ${ }^{1}$, O.Sorensen ${ }^{1}$, W. L.Suarez-Pinzon ${ }^{1}$, R. F.Power ${ }^{2}$, R. V.Rajotte ${ }^{3}$, R.C. Bleackley ${ }^{4}$ \\ ${ }^{1}$ Department of Medicine, University of Alberta, Edmonton, Alberta, Canada \\ ${ }^{2}$ Department of Pathology, University of Alberta, Edmonton, Alberta, Canada \\ ${ }^{3}$ Department of Surgery, University of Alberta, Edmonton, Alberta, Canada \\ ${ }^{4}$ Department of Biochemistry, University of Alberta, Edmonton, Alberta, Canada
}

\begin{abstract}
Summary The injection of complete Freund's adjuvant into diabetic nonobese diabetic (NOD) mice at the time of syngeneic islet transplantation prevents monocytic/lymphocytic cell infiltration into the islet graft, Beta-cell destruction, and autoimmune diabetes recurrence. We have used semiquantitative reverse transcriptase-polymerase chain reaction analysis to examine and compare cytokine mRNA expression profiles in islet grafts from complete Freund's adjuvantinjected and control NOD mice. Interleukin 10 mRNA expression was significantly increased whereas interleukin 2 and interferon gamma mRNA levels were significantly decreased in islet grafts from complete Freund's adjuvant-injected mice compared to control mice. Levels of mRNA for interleukin 1 beta, inter-
\end{abstract}

leukin 4 , and tumour necrosis factor alpha were not significantly different in islet grafts from complete Freund's adjuvant-injected and control mice. These findings suggest that a Th1 subset of lymphocytes and their cytokine products, interleukin 2 and interferon gamma, may be involved in the rejection of syngeneic islet grafts and diabetes recurrence in NOD mice, and that the protective effect of complete Freund's adjuvant may result from the induction of interleukin 10 production and consequent down-regulation of Th1 cells and cytokines in the islet graft. [Diabetologia (1994) 37: 833-837]

Key words Nonobese diabetic mouse, islet transplantation, cytokines.
Diabetes mellitus in NOD mice results from immunecell-mediated destruction of the insulin-producing Beta cells in the islets of Langerhans. This autoimmune process can be suppressed by a variety of microbial agents, ranging from viruses to bacterial, fungal and mycobacterial preparations - including CFA and bacillus Calmette-Guérin (BCG) [1]. CFA can prevent both

Received: 25 January 1994

and in revised form: 4 March 1994

Corresponding author: Dr. A. Rabinovitch, 430 Heritage Medical Research Centre, University of Alberta, Edmonton, Alberta T6G 2 S2, Canada

Abbreviations: NOD, Nonobese diabetic mouse; CFA, complete Freund's adjuvant; PCR, polymerase chain reaction; IL, interleukin; TNF $\alpha$, tumour necrosis factor alpha; IFN $\gamma$, interferon gamma; BBrat, bio-breeding rat; PBS, phosphate buffered saline the inductive and the effector phases of the autoimmune response leading to Beta-cell destruction and diabetes, and the protective effects of CFA have been related to induction of $T$ regulatory (suppressor) cells [1] and to a state of T-cell dormancy [2]. Since T-cell responses are modulated by cytokines, and since a variety of cytokines have been reported to protect against diabetes appearance in NOD mice [3], we questioned whether the protective effect of CFA against diabetes in NOD mice could be explained by effects on cytokine production. To test this hypothesis, we compared levels of cytokine mRNA in syngeneic islet grafts transplanted into diabetic NOD mice protected from graft rejection by CFA administration [4] and in syngeneic islet grafts transplanted into control (non-protected) diabetic NOD mice. 

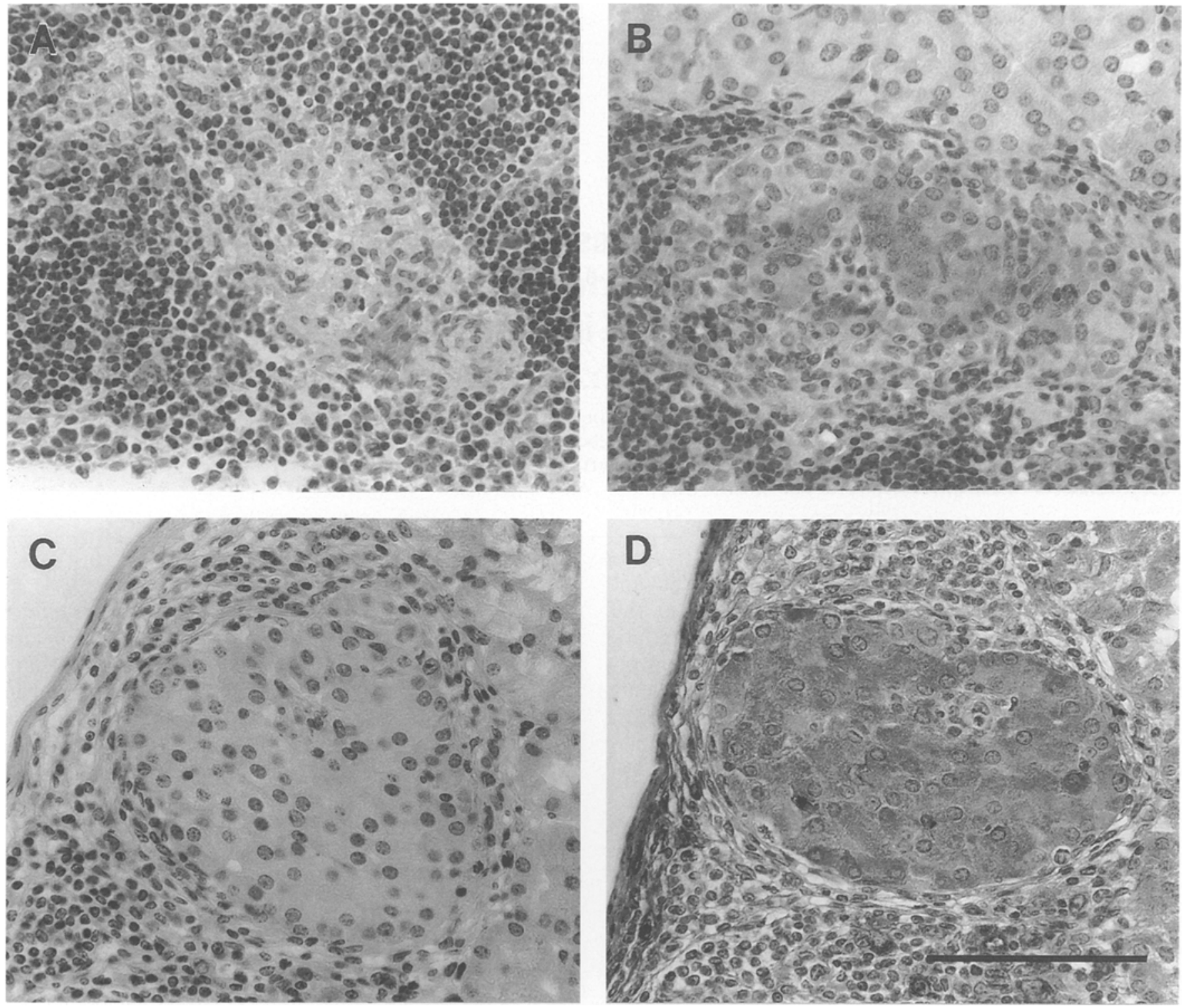

Fig. 1A-D. Photomicrographs of islet grafts transplanted under the renal capsule in diabetic NOD mice and removed 10 days post transplantation (bar scale, $100 \mu \mathrm{m}$ ). Sections were stained with haematoxylin-eosin (A and $\mathbf{C}$ ) and with anti-insulin antibody and immunoperoxidase-conjugated second antibody (B and $\mathbf{D})$. $\mathbf{A}$ and $\mathbf{B}$ : sections of an islet graft from a saline-injected (control) NOD mouse. The islet is infiltrated by mononuclear cells (A) and insulin-staining Beta cells are reduced in number (B). C and D: sections of an islet graft from a CFA-injected NOD mouse. Mononuclear cells are seen adjacent to but not infiltrating into the islet $(\mathbf{C})$ and insulin-staining Beta cells are well preserved (D)

\section{Materials and methods}

Animals. NOD mice were purchased from Taconic Farms Inc. (Germantown, N. Y., USA) and were maintained and fed under pathogen-free conditions. Diabetes (blood glucose $>11.1 \mathrm{mmol} / \mathrm{l}$ ) occurred in female NOD mice beginning at age 13 weeks and reached $80-100 \%$ by age 30 weeks. Diabetic mice

were maintained by daily injections of ultralente beef and pork insulin (1 IU/100 g body weight) until the day before receiving islet transplantation. Female diabetic NOD mice, age 2224 weeks, with diabetes duration of 2-6 weeks served as islet graft recipients. Islet donors were male NOD mice, age 5 6 weeks. All mice were cared for according to the guidelines of the Canadian Council on Animal Care.

Islet transplants. Islets were isolated by stationary collagenase digestion of the pancreas and Ficoll density gradient isolation followed by hand picking of the islets. Five hundred freshly-isolated islets (from $\approx$ four donor mice) were transplanted under the left renal capsule in each diabeticNOD recipient mouse, according to a previously described procedure [4]. Transplantation was considered technically successful if the non-fasting blood glucose returned to normal $(<8.8 \mathrm{mmol} / \mathrm{l})$ within $2-3$ days. Tail vein blood glucose was monitored daily post transplantation, by using an Accu-Chek IIm glucose monitor (Boehringer Mannheim, Laval, Quebec, Canada). Islet graft rejection was diagnosed by return of hyperglycaemia ( $>15 \mathrm{mmol} / \mathrm{l}$ ) on two consecutive days.

CFA administration. CFA (Difco, Detroit, Mich., USA) was injected $(50 \mu \mathrm{l})$ into each hind footpad at the time of islet trans- 
plantation, and control mice were similarly injected with PBS at the time of islet transplantation.

Histopathology. Islet grafts were removed from CFA-injected and control NOD mice at 10 days after islet transplantation. The grafts were fixed in $10 \%$ buffered formalin, embedded in paraffin, sectioned at $4.5 \mu \mathrm{m}$, and stained with haematoxylin-eosin, and with anti-insulin antibody (Dako Corp., Corpinteria, Calif., USA) using an immunoperoxidase technique. Coded slides were read by light microscopy.

Cytokine mRNA determinations. Islet grafts were removed from CFA-injected and control NOD mice at 10 days after islet transplantation. RNA was extracted from the grafts by a modification of the guanidinium thiocyanate method of Chirgwin et al. [6]. Specifically, tissues were homogenized in $4 \mathrm{~mol} / \mathrm{l}$ guanidinum thiocyanate solution containing $17 \mathrm{mmol} / 1$ sodium $\mathrm{N}$-lauroylsarcosine, $25 \mathrm{mmol} / \mathrm{l}$ sodium citrate, $0.1 \mathrm{~mol} / \mathrm{l}$ 2-mercaptoethanol and $0.1 \%$ Antifoam A, 30\% (Sigma, St. Louis, Mo., USA), then precipitated with ethanol, pelleted and re-extracted twice with $8 \mathrm{~mol} / 1$ guanidine hydrochloride: $0.5 \mathrm{~mol} / 1$ EDTA (19: 1). Following pelleting and drying, samples were extracted twice with phenol:chloroform (1:1) and precipitated with ethanol. Pelleted RNA samples were covered with $70 \%$ ethanol and stored at $70^{\circ} \mathrm{C}$. cDNA synthesis was carried out on $5 \mu \mathrm{g}$ of total RNA with a Superscript Reverse Transcriptase Kit (Gibco BRL, Burlington, Ontario, Canada), using oligo (dT) ${ }_{12-18}$ and M-MLV reverse transcriptase $(20 \mathrm{U})$ in a $1.5 \mathrm{~h}$ incubation at $37^{\circ} \mathrm{C}$. PCR amplification of cDNA was carried out in a $20-\mu \mathrm{l}$ reaction on $1 \mu \mathrm{l}$ of cDNA (the equivalent of $20 \mathrm{ng}$ of total RNA) and 1:2,1:10, and 1: 20 dilutions, in the presence of $80 \mathrm{ng}$ of each primer, $0.25 \mathrm{mmol} / \mathrm{l}$ of each dNTP, $2.5 \mu \mathrm{Ci}$ of $\left[\alpha_{-}{ }^{32} \mathrm{P}\right] \mathrm{dCTP}$ (3000 Ci/mmol, Dupont, Mississauga, Ontario, Canada), $1 \mathrm{U}$ of ampliTaq (Perkin Elmer Cetus, Norwalk, Conn., USA), and $3 \mathrm{mmol} / 1 \mathrm{Mg}^{2+}$. Samples were amplified through 40 cycles at $94^{\circ} \mathrm{C}$ for $20 \mathrm{~s}, 60^{\circ} \mathrm{C}$ for $20 \mathrm{~s}$, and $72^{\circ} \mathrm{C}$ for $30 \mathrm{~s}$ in a Gene Amp PCR System 9600 (Perkin Elmer Cetus). The sequences of the specific oligonucleotide primer pairs, $5^{\prime}$ and $3^{\prime}$, are as follows: IL- $1 \beta$ : ATGGCAACTGTTCCTGAACTCAAC and CAGGACAGCTATAGATTCTTTCCTTT; IL-2: CCTGAGCAGGATGGAGAATTACAG and CCTATGTGTTGTAAGCAGGAGGTAC; IL-4: GTCTCTCGTCACTGCCGCACAGAGCTATTG and CATGATGCTCTTTAGGCTTTCCAGGAAGTC; IL-10: AGCTGGACAACATACTGCTAACC and TCATTCATGGCCTTGTAGACAC; TNF $\alpha$ : CTTAGACTTTGCGGAGTCCG and ACAGTCCAGGTCACTGTCCC; IFN $\gamma$. CGCTACACACTGCATCTTGG and GGCTGGATTCCGGCAACA; cyclophilin: GACAGCAGAAAACTTTCGTGC and TCCAGCCACTCAGTCTTGG. The PCR reaction was electrophoresed on $1.5 \%$ agarose gels and transfered to nylon membranes. Bands detected by autoradiography were cut out of the nylon membrane and the incorporated ${ }^{32} \mathrm{P}$ was counted. The values obtained for each cytokine PCR product were normalized as a percent of ${ }^{32} \mathrm{P}$ incorporated in cyclophilin PCR product. Under the conditions used, there was a linear relationship between the cDNA amount (serial dilutions) subjected to PCR amplification and the PCR product signal. Samples of cDNA to be compared (from CFA-injected and control mice) were amplified in the same PCR run. This assay is semiquantitative, i.e. it allows for the determination of the relative amount of mRNA for a given cytokine in different samples, however it does not allow for a precise determination of the amount of mRNA present in each sample. Similar semiquantitative PCR assays have been used to analyse cytokine mRNA expression in other conditions [6].

\section{Statistical analysis}

Data are presented as means \pm SEM. Statistical comparisons between groups were made by Student's unpaired $t$-test, with $p$ less than 0.05 considered significant.

\section{Results}

In a previous study, we reported that syngeneic islet grafts in diabetic NOD mice were rejected beginning 10 days after transplantation and median graft survival time was only 17 days compared to more than 107 days in CFA-injected diabetic NOD mice receiving islet grafts [4]. Therefore, in the present study, we harvested islet grafts from control and CFA-injected NOD mice at 10 days after islet transplantation, i. e. before the earliest time of graft rejection and diabetes recurrence in the control mice. Histological examination of the islet grafts from control mice showed massive infiltration of the islets by mononuclear leucocytes and loss of insulin-containing Beta cells (Fig. 1, A and B). In contrast, islet grafts from CFA-injected mice were not infiltrated by mononuclear leucocytes, rather these cells remained outside the islet and insulin-containing Beta cells were well preserved (Fig. 1, C and D). The photomicrographs in Figure 1 are representative of four control and four CFA-injected mice, and the findings are similar to those previously reported [4].

To determine if these histological differences in the islet grafts of CFA-injected and control mice might be related to differences in cytokines produced by the mononuclear leucocytes in the grafts, we compared levels of cytokine mRNA in the islet grafts by using a semiquantitative PCR assay. Levels of cytokine mRNA in islet grafts from CFA-injected and control mice are shown in Figure 2. Average mRNA levels for IL-2 $(p<0.02)$ and IFN $\gamma(p<0.05)$ were significantly decreased in grafts from CFA-injected mice. TNF $\alpha$ mRNA was also decreased in grafts from CFA-treated mice, however this did not reach statistical significance $(p<0.1)$. IL-1 $\beta$ and IL-4 mRNA levels were not different in grafts from CFA-injected and control mice. The level of IL-10 mRNA was significantly increased in islet grafts from CFA-injected mice $(p<0.02)$.

\section{Discussion}

In this study, we confirmed our previous observation that injection of CFA at the time of syngeneic islet transplantation in diabetic NOD mice prevents infiltration of the islet graft by mononuclear leucocytes, Betacell destruction, and diabetes recurrence [4]. Islet grafts in CFA-treated NOD mice revealed a peri-islet accumulation of mononuclear leucocytes that did not infiltrate into the islet graft and Beta cells remained intact. Notably, the expression of cytokine genes (mRNA) was different in islet grafts from CFA-treated mice and 

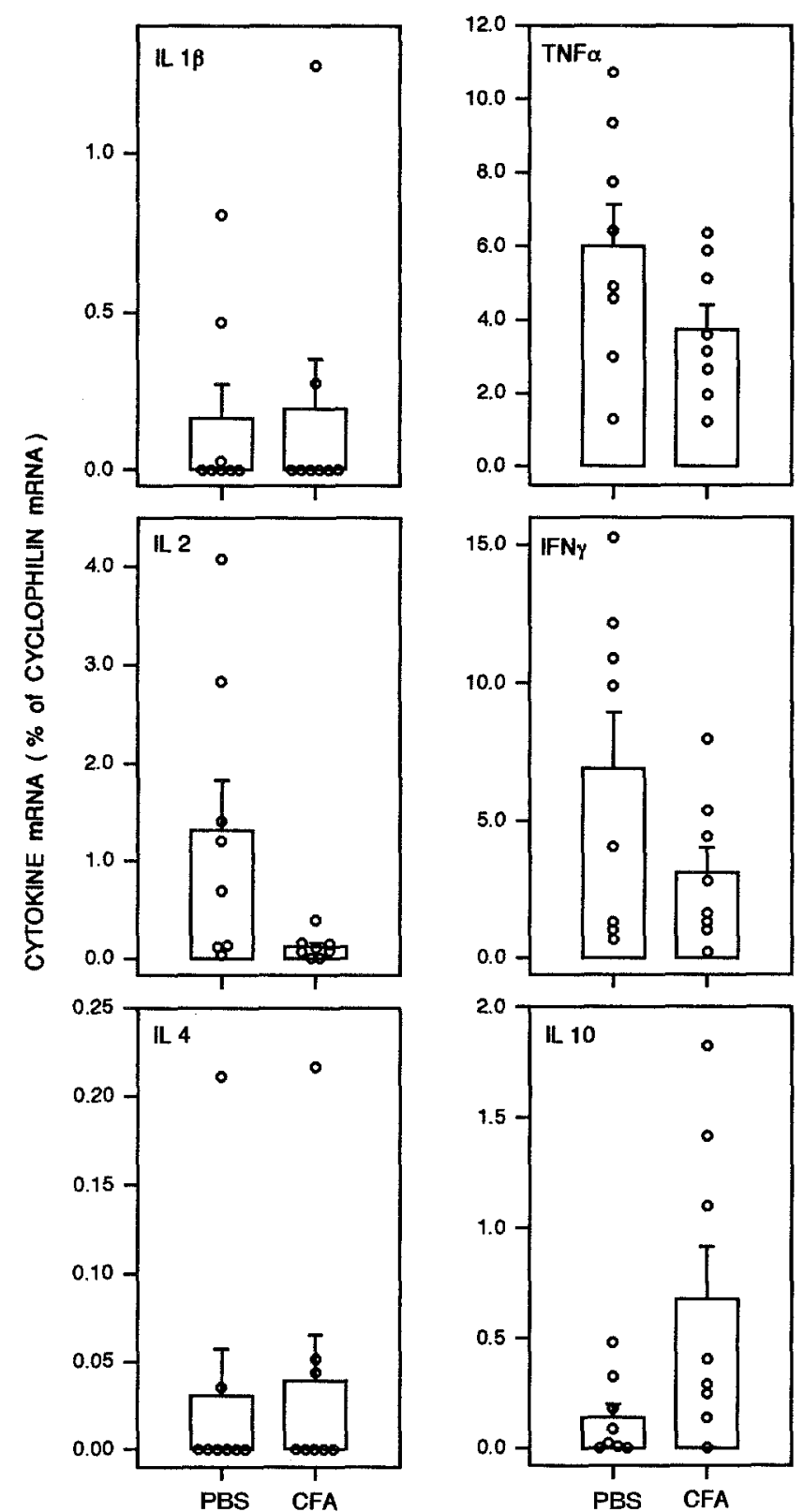

Fig. 2. Levels of expression of mRNA for IL- $1 \beta$, TNF $\alpha$, IL-2, IFN $\gamma$, IL-4, and IL-10 in islet grafts at 10 days post transplantation in PBS-injected (control) and CFA-injected NOD mice. Cytokine mRNA levels were determined by PCR assay, and are expressed as $100 \% \times$ the ratio of the cytokine PCR product to the cyclophilin PCR product amplified from cDNA from the same islet graft sample. Cytokine mRNA values are shown for islet grafts from individual PBS-injected NOD mice $(n=8)$ and CFAinjected NOD mice $(n=8)$ and mean values \pm SEM for the two groups of mice are shown as columns

islet grafts from control mice. Thus, IL-10 mRNA expression was increased whereas IL-2 and IFN $\gamma$ mRNA levels were decreased in islet grafts from CFA-injected mice compared to control mice. Levels of mRNA for IL-1 $\beta$, IL-4 and TNF $\alpha$ were not significantly different in grafts from CFA-injected and control mice.

The combination of increased expression of IL-10 mRNA together with decreased expression of IL-2 and IFN $\gamma$ mRNA is noteworthy since IL-10 inhibits proliferation and cytokine production (IL-2 and IFN $\gamma$ ) by antigen-stimulated helper T cells of the Th1 subset [7]. IL-10 also inhibits release of inflammatory cytokines (IL-1, IL-6, IL-8, TNF $\alpha$ ) by monocytes/macrophages, increases production of IL-1 receptor antagonist, and inhibits MHC class II expression, which leads to prevention of T-cell activation and reduces further the release of inflammatory cytokines [7]. These activities of IL-10 suggest that the functions of endogenous IL-10 are to down-regulate inflammatory responses mediated by monocytes/macrophages and their cytokine products, as well as to down-regulate cell-mediated immune responses triggered by Th1 cells and their cytokine products (IL-2 and IFN $\gamma$ ). Therefore, our findings of increased IL-10 mRNA together with decreased IL-2 and IFN $\gamma$ mRNA levels in islet grafts of CFA-injected mice suggest that the adjuvant may have stimulated IL-10 production and this, in turn, could have down-regulated IL-2 and IFN $\gamma$ production by Th1 cells, leading to suppressed immune/inflammatory responses to the islet graft, reduced graft invasion by monocytic and lymphocytic cells, and prevention of islet Beta-cell destruction and diabetes recurrence.

Our proposal that CFA-induced $\mathrm{IL}-10$ production in the islet graft may have suppressed islet infiltration and diabetes recurrence is supported by a recent study reporting that chronic IL-10 administration significantly decreases insulitis severity and the incidence of spontaneous diabetes in NOD mice [8]. Also, the peri-islet infiltrate we observed in islet grafts of CFA-treated mice is compatible with actions of IL-10. Thus, transgenic expression of $\Pi \mathrm{L}-10$ by islet Beta cells in mice leads to pronounced vascular endothelial cell changes and leucocyte extravasation into the pancreas without cellular infiltration of the islets, Beta-cell damage and diabetes [9]. In addition, our finding that expression of IL-10 mRNA in the islet graft correlated with graft survival is similar to the report that IL-10 mRNA expression in the central nervous system of mice with experimental autoimmune encephalomyelitis (an animal model of multiple sclerosis) correlates with recovery from this autoimmune disease [6].

The present findings of decreased $\mathrm{mRNA}$ levels of Th1 cell-derived cytokines (IL-2 and IFN $\gamma$ ) in islet grafts of CFA-injected NOD mice are in general agreement with a recent report that IFN $\gamma$-producing T cells are decreased and IL-4-producing T cells are relatively increased in syngeneic pancreatic islet "sentinel" grafts in NOD mice protected against spontaneous diabetes by CFA injection [10]. In our study, IL-4 gene expression was not increased in the CFA-protected grafts, rather there was increased expression of another Th2 cell-derived cytokine, IL-10. However, IL-10 is a product of macrophages as well as of Th2 cells [7], and the present study does not identify the cell source of IL-10 up-regulated by CFA. 
The present findings are in agreement with other studies regarding the roles of cytokines in the pathogenesis of autoimmune diabetes [reviewed in 3]. Thus, IFN $\gamma$ has been assigned a cytotoxic role, based on the following observations: IFN $\gamma$ is cytotoxic to islet cells in vitro; transgenic mice expressing IFN $\gamma$ under control of the insulin promoter develop insulitis and diabetes; and antibodies to IFN $\gamma$ prevent diabetes development in NOD mice and BB rats. Evidence for involvement of IL-2 in the development of diabetes is ambivalent. IL-2 transgenic mice develop insulitis, however this progresses to diabetes only when other transgenes (MHC class I and corresponding T-cell antigen receptor molecules) are expressed in the mice. Involvement of IL-2 in diabetes development is suggested by the observation that diabetes in NOD mice can be blocked by treatment with a diphtheria toxin-related IL-2 fusion protein targeted to the IL-2 receptor. On the other hand, chronic treatment of NOD mice with IL-2 significantly decreases insulitis and diabetes incidence. Also, IL-2 administration decreases diabetes incidence in a $\mathrm{BB}$ rat subline with a high diabetes risk, however, diabetes is increased in a $\mathrm{BB}$ rat subline with a low diabetes risk.

In conclusion, the present findings point to an immune process involving Th1 cells and their cytokine products, IL- 2 and IFN $\gamma$, in the Beta-cell destructive infiltrate associated with rejection of a syngeneic islet graft and autoimmune diabetes recurrence in NOD mice. Furthermore, IL-10 is identified as a possible suppressor of this Th1-cell-mediated immune process involved in islet Beta-cell destruction and diabetes recurrence.

Acknowledgements. This work was supported by a program grant from the Juvenile Diabetes Foundation International; the MacLachlan Fund of the University of Alberta Hospitals, and the Muttart Diabetes Research and Training Centre. The authors thank Drs. T. Mosmann and S. Narula for helpful suggestions, and Ms. A.Fu, Mr. M. Dhalla and Ms. D.Dixon for technical assistance.

\section{References}

1. Singh B, Rabinovitch A (1993) Influence of microbial agents on the development and prevention of autoimmune diabetes. Autoimmunity 15:209-213

2. Ulaeto D, Lacy PE, Kipnis DM, Kanagawa O, Unanue ER (1992) A T-cell dormant state in the autoimmune process of nonobese diabetic mice treated with complete Freund's adjuvant. Proc Natl Acad Sci USA 89: 3927-3931

3. Rabinovitch A (1993) Roles of cytokines in IDDM pathogenesis and islet $\beta$-cell destruction. Diabetes Reviews 1: $215-240$

4. Wang T, Singh B, Warnock GL, Rajotte RV (1992) Prevention of recurrence of IDDM in islet-transplanted diabetic NOD mice by adjuvant immunotherapy. Diabetes 41 : 114 117

5. Chirgwin JM, Przybyla AE, MacDonald RJ, Rutter KT (1979) Isolation of biologically active ribonucleic acid from sources enriched in ribonuclease. Biochemistry 18: 5294 5299

6. Kennedy MK, Torrance DS, Picha KS, Mohler KM (1992) Analysis of cytokine mRNA expression in the central nervous system of mice with experimental autoimmune encephalomyelitis reveals that IL-10 mRNA expression correlates with recovery. J Immunol 149: 2496-2505

7. Moore KV, O'Garra A, de Waal Malefyt R, Vieira P, Mosmann TR (1993) Interleukin-10. Ann Rev Immunol 11: 165190

8. Pennline KJ, Roque-Gaffney E, Monahan M (1994) Recombinant human IL-10 (rHuIL-10) prevents the onset of diabetes in the nonobese diabetic (NOD) mouse. Clin Immunol Immunopath 71: 169-175

9. Wogensen L, Huang X, Sarvetnick N (1993) Leukocyte extravasation into the pancreatic tissue in transgenic mice expressing interleukin 10 in the islets of Langerhans. J Exp Med 178: 175-185

10. Shehadeh N, LaRosa F, Lafferty KJ (1993) Altered cytokine activity in adjuvant inhibition of autoimmune diabetes. $\mathbf{J}$ Autoimmunity 6:291-300 